\title{
SENSIBILIDAD A LAS SENSACIONES CORPORALES E HIPOCONDRÍA
}

\author{
AMPARO BELLOCH ${ }^{1}$, ESTHER AYLLÓN ${ }^{1}$, M. PILAR MARTÍNEZ ${ }^{2}$, CLAUDIA \\ CASTAÑEIRAS ${ }^{1}$ y M. ÁNGELES JIMÉNEZ ${ }^{3}$ \\ ${ }^{1}$ Universidad de Valencia \\ ${ }^{2}$ Universidad de Granada \\ ${ }^{3}$ Universidad de Murcia
}

(Recibido el 8 de febrero de 1998)

\begin{abstract}
Se ha postulado que la tendencia a experimentar las sensaciones somáticas de un modo intenso y perturbador es un elemento característico de la hipocondría y forma parte de un hipotético estilo perceptivo amplificador, si bien su presencia no se restringe a esta entidad y constituye un síntoma inespecífico de otros trastornos mentales que cursan con sintomatologia física. El objetivo del presente estudio ha sido examinar la presencia de dicho estilo amplificador en la población general mediante la aplicación a una muestra de 457 sujetos sanos, de la Escala de Amplificación Somatosensorial (SAS). Los sujetos cumplimentaron además la subescala de Conciencia Corporal Privada del Cuestionario de Conciencia Corporal, el Indice Witheley, la Escala de Preocupación por la Enfermedad, la escala de Hipocondría del MMPI, el Inventario de Ansiedad Estado-Rasgo, STAI, y el Inventario de Depresión de Beck. Los resultados obtenidos indican que la SAS posee niveles satisfactorios de consistencia interna, validez de contenido y validez concurrente. Además, puntuaciones superiores a 21 identifican con razonable seguridad personas amplificadoras entre la población normal.
\end{abstract}

Palabras clave: Hipocondría, amplificación somato-sensorial, auto-consciencia corporal, sensaciones corporales.

\section{Bodily sensitivity and hypochondriasis}

The propensity to experience the somatic sensations as disturbing and noxious has been postulated as being a pathogenic factor in hypochondriasis. This somatic amplification style may also be a non-specific characteristic of some mental disorders with physical symptomatology. The aim of the current study has been to examine the somatic amplification style in the normal population. With this purpose 457 subjects completed the following instruments: Somatosensory Amplification Scale (SAS); Private Body Consciousness scale (Body Consciousness Questionnaire); MMPI Hypochondriasis scale; State-Trait Anxiety Inventory, and Beck Depression Inventory. Our results showed a satisfactory internal consistency, content validity and concurrent validity of the SAS. Moreover, total scores greater than 21 on this scale could be considered as a reliable cut-off point to identify persons who tend to amplify their somatic sensations.

Key words: Hyponchondriasis, Somatosensory Amplification Scale, body consciousness, body sensitivity.

\section{INTRODUCCIÓN}

\section{La hipocondría constituye una categoría nosológica con entidad propia desde los}

Correspondencia: Amparo Belloch. Dpto. de Personalidad, Evaluación y Tratamiento Psicológicos, Facultad de Psicología. Universidad de Valencia, Avda. Blasco Ibáñez 21. 46010 Valencia. Correo-e: belloc@ uv.es inicios de la caracterización y el diagnóstico psicopatológicos. Además, algunos instrumentos psicométricos clásicos, como el MMPI de Hathaway y MacKinley, ampliamente utilizado y conocido desde su primera publicación en 1943, incluyeron la hipocondría entre los síndromes identificables de trastorno mental. Pero paradójicamente este reconoci- 
miento no iba asociado a una investigación sistemática de las características supuestamente asociadas a dicha entidad ni, consecuentemente, al planteamiento de modelos teóricos que pudieran ofrecer explicaciones plausibles, empíricamente verificables, sobre su etiopatogenia y mantenimiento. Sin embargo, en las dos últimas décadas estamos asistiendo a una cierta proliferación de modelos explicativos que intentan dar cuenta de los mecanismos que subyacen a la génesis y mantenimiento de la hipocondría y que asientan en paradigmas teóricos tan dispares como el físiológico, el psicosocial y el cognitivo.

Si bien es cierto que la mayoría de las propuestas actuales reconocen que la hipocondría no obedece a una causa única, sino a un proceso interactivo de variables de diversa índole, cada una de ellas subraya la importancia de un elemento frente a los demás. Así, por ejemplo, desde una perspectiva cognitiva se ha considerado que la hipocondría es producto, entre otras cosas, de alteraciones localizadas en el ámbito perceptivo y/o atencional. En esta línea, Barsky y colaboradores (Barsky, 1979, 1992; Barsky, Goodson, Lane y Cleary, 1988; Barsky y Klerman, 1983; Barsky y Wyshak, 1990) han planteado que la hipocondría puede ser entendida y explicada sobre la base de lo que denominan un «estilo somático amplificador». Desde esta perspectiva, se postula que los sujetos hipocondríacos amplifican las sensaciones somáticas y viscerales, es decir, tienden a experimentar tales sensaciones como más intensas, nocivas y perturbadoras que los no hipocondríacos. Los síntomas susceptibles de amplificación en estas personas cubren un amplio espectro (Barsky, 1992): sensaciones fisiológicas y anatómicas normales (p.ej., movimientos peristálticos intestinales, hipotensión postural, variaciones en la tasa cardíaca), disfunciones benignas y enfermedades leves (p.ej., zumbidos pasajeros, contracciones nerviosas de los párpados, sequedad de la piel), y concomitantes viscerales o somáticos de un estado emocional (p.ej., la activación fisiológica que acompaña a la ansiedad).

El estilo perceptivo amplificador característico del hipocondríaco incluye tres elementos (Barsky, 1992; Barsky et al., 1988): (1) hipervigilancia corporal, que conlleva un aumento del auto-escrutinio y del enfoque de la atención en sensaciones corporales desagradables; (2) tendencia a seleccionar y centrarse en ciertas sensaciones relativamente infrecuentes y/o tenues; y (3) propensión a valorar las sensaciones somáticas y viscerales como anómalas, patológicas e indicadoras de enfermedad, en vez de como normales.

Pero además, según Barsky (1992), el concepto de amplificación resulta de utilidad para comprender diversas condiciones clínicas, tanto psicológicas como físicas, que se caracterizan por presentar quejas somáticas desproporcionadas con relación al posible daño orgánico existente. Desde esta perspectiva, se propone que la amplificación juega un role patogénico en la hipocondría pero, a la vez, constituye un síntoma inespecífico de otros trastornos mentales que cursan con síntomas físicos (p.ej., el trastorno por angustia o los estados depresivos), así como en los procesos más o menos transitorios y no necesariamente patológicos de somatización, tales como los que se producen como consecuencia de la exposición a acontecimientos vitales estresantes. A la vez podría explicar, al menos en parte, las diferencias en la intensidad con la que experimentan los síntomas personas que padecen una misma enfermedad médica (p.ej., artritis reumatoide, asma, arritmia cardíaca, enfermedades del tracto digestivo, etc.).

En consecuencia con lo que se acaba de exponer, parece lícito suponer que la amplificación no constituye una entidad 
o síntoma del tipo "todo-o-nada" sino, muy al contrario, se puede conceptuar como una dimensión continua, fuente de diferencias individuales, en la que los hipocondríacos ocuparían los lugares más extremos del continuo. Partiendo de esta consideración, nos planteamos analizar la presencia de esta supuesta dimensión psicológica en un grupo relativamente amplio de personas sin patología mental ni médica constatables, mediante la aplicación de la Escala de Amplificación Somatosensorial (Somatosensory Amplification Scale, SAS) diseñada por Barsky, Wyshak y Klerman (1990) para evaluar el constructo de amplificación. Para ello se examinan además las propiedades psicométricas básicas de esta escala (consistencia interna, validez de contenido y validez concurrente).

\section{MATERIAL Y MÉTODO}

\section{Muestra}

En el estudio han participado 457 personas, de las que 338 eran mujeres y 119 varones. El rango de edad osciló entre los 19 y 60 años (media \pm DT $=28,75 \pm 11,98$ ). Residían en un medio urbano (Valencia, Murcia), o en poblaciones próximas al área metropolitana. Los criterios de inclusión en el estudio fueron: ausencia de enfermedad actual diagnosticada (médica o mental), ausencia de historia de alcoholismo o abuso de sustancias, adecuada comprensión lectora, y rango de edad entre 19 y 60 años. Los sujetos cumplimentaron la adaptación española de los instrumentos, junto con información demográfica básica (edad, sexo, estado civil, nivel cultural y profesión) de forma anónima y voluntaria. El $63 \%$ de los sujetos eran estudiantes universitarios de los últimos cursos y el restante $37 \%$ pertenecía a diversos niveles profesionales. El $71 \%$ de los participantes estaban solteros, mientras que el $25 \%$ eran casados, y el $4 \%$ restante estaba separado. En la Tabla 1 se recogen los datos demográficos más relevantes.

En el caso de los estudiantes el pase de los cuestionarios se realizó de manera colectiva, mientras que los no estudiantes los completaron de forma individual. Todos los participantes fueron debidamente informados de la finalidad del estudio y dieron su consentimiento voluntario para participar en el mismo.

\section{Instrumentos}

Escala de Amplificación Somatosensorial (SAS) (Barsky, Wyshak y Klerman, 1990). La SAS fue construida con el propósito de evaluar la sensibilidad a las sensaciones corporales benignas que son molestas pero que no constituyen síntomas típicos de enfermedad. Este instrumento está compuesto por 10 ítems, respecto a los cuales la persona ha de decidir, de acuerdo con una escala Likert de 5 puntos que oscila desde 1 ("nada») hasta 5 ( (muchísimo»), el grado en que cada uno de ellos «le caracterizan en general». El rango posible de puntuaciones oscila entre 10 y 55 .

Cuestionario de Conciencia Corporal (Body Consciousness Scale, BCQ, Miller, Murphy y Buss, 1981). Este instrumento mide aspectos de la conciencia corporal pública y privada y está compuesto de 3 subescalas, de las que en el presente estudio se seleccionó una de ellas por su adecuación para los objetivos del mismo: la escala de Conciencia Corporal Privada (CCP). Esta subescala contiene 5 ítems medidos con una escala Likert de 5 puntos que oscila desde 0 («nada») hasta 4 ( «muchísimo»), de los cuales eliminamos uno debido a que coincidía con un ítem de la SAS.

Cuestionario de Conducta de Enfermedad (Illness Behaviour Questionnaire, 
$I B Q$, Pilowsky y Spence, 1983). El IBQ permite, según sus autores, medir las actitudes que indican modos desadaptativos o inapropiados de responder al propio estado de salud. La escala se compone de 62 ítems a los que se debe responder según dos alternativas de respuesta ("Sí» / «No»). Para la presente investigación hemos utilizado únicamente una subescala de este instrumento, el índice Whiteley (IW), compuesto por 14 items diseñados para explorar actitudes hipocondríacas. Se ha utilizado la versión en castellano de Ballester y Botella (1993).

Escala de Preocupaciones por la Enfermedad (EPE) (Illness Concerns Scale, Wesner y Noyes, 1991). Se trata de un instrumento diseñado para evaluar la fobia a la enfermedad, tanto en sus manifestaciones cognitivas (i.e., preocupación, rumiación) como en las comportamentales (i.e, evitación). Incluye 18 enunciados, algunos de los cuales fueron extraídos del IBQ, que debe ser valorado en una escala Likert de cinco pasos (desde 0 = "nada en absoluto", hasta $4=$ "muchísimo»). Se ha utilizado la versión previamente adaptada al castellano en una muestra de 300 adultos (Jiménez, Castañeiras, Martínez, Ayllón y Belloch, 1997).

Escala de Hipocondriasis (Hs) del Inventario de Personalidad Multifásico de Minnesota (MMPI) (Hathaway y Mckinley, 1967). La escala está formada por 33 enunciados con dos alternativas de respuesta ("verdadero" o "falso"), y se ha utilizado ampliamente como medida de síntomas somáticos.

Inventario de Ansiedad Estado-Rasgo (State-Trait Anxiety Inventory, STAI; Spielberger, Gorsuch y Lushene, 1970). Este instrumento está compuesto por dos escalas separadas de auto evaluación que miden aspectos conceptuales independientes de ansiedad: la Ansiedad Estado (A-E) y la Ansiedad Rasgo (A-R). En conjunto, el STAI consta de 40 ítems (20 para cada subescala), que deben ser valorados por el individuo sobre la base de cuatro alternativas de respuesta (de 0 a 3 ). Se ha utilizado la versión española del cuestionario (Seisdedos, 1988)

Inventario de Depresión de Beck (Beck's Depression Inventory, BDI; Beck, Rush, Shaw y Emery, 1979). Se trata de un instrumento integrado por 21 grupos de 4 enunciados cada uno que describen síntomas depresivos (p. ej., tristeza, lloro, indecisión, pensamientos de suicidio) de los que el sujeto tiene que elegir el que considere que describe mejor los sentimientos que ha experimentado durante la última semana. La puntuación de los ítems oscila en un intervalo de 0 a 3 . Para este estudio hemos utilizado la versión española que aparece en la traducción castellana del libro de Beck et al. (1979) Terapia Cognitiva de la Depresión, con las correcciones de errores correspondientes a los ítems 10 y 20.

\section{Análisis Estadísticos}

Para los análisis de fiabilidad (consistencia interna) de la SAS se calculan los estadísticos $\alpha$ de Cronbach, Guttman, y Spearman-Brown (para examinar la correlación entre las dos mitades del cuestionario). Además se calcularon las correlaciones entre cada ítem y el total de la escala, y entre cada ítem y la puntuación total corregida (esto es, la puntuación total sin incluir el ítem en cuestión). La validez de contenido se examinó mediante análisis factorial de componentes principales con rotación varimax, eigenvalue $<1$, y scree test para la determinación de la estructura factorial más interpretable. Para la inclusión de un ítem en un factor se exige una saturación mínima de 0,40 . La validez concurrente se analiza mediante correlaciones Spearman. Las comparaciones entre grupos se realizan mediante ANOVAs ( 3 o más gru- 
pos) o pruebas t para muestras independientes ( 2 grupos). Los análisis a posteriori de los valores de $\mathrm{F}$ obtenidos se analizaron mediante la prueba de Bonferroni. Para examinar cuáles de las variables contempladas en el estudio pueden predecir la puntuación total en la SAS se realizó un análisis de regresión múltiple de pasos sucesivos. La variable dependiente fue la puntuación total en la SAS y las variables independientes que se introdujeron fueron aquellas que mostraron correlaciones significativas (Spearman) con la SAS. La ratio variables/sujetos mínima exigida para este tipo de análisis $(1 / 10)$ fue ampliamente superada en nuestro caso (12 variables/457 sujetos). Antes de la aplicación de pruebas paramétricas se constató la existencia de distribución normal en las puntuaciones, con el fin de normalizarlas en caso de que fuera necesario. El valor mínimo de significación estadística exigido fue de
0,05 . Todos los análisis se realizaron con el paquete estadístico SPSS 7.0.

\section{RESULTADOS}

En la Tabla 1 se recogen los principales resultados obtenidos en los cuestionarios (medidas de tendencia central), así como los relacionados con las características socio-demográficas de la muestra. Hay que señalar el hecho de que todas las puntuaciones de los cuestionarios se ajustaron a una distribución normal.

Los resultados obtenidos en el análisis de consistencia interna de la SAS arrojaron un valor estandarizado de $\alpha=0,80$. Este valor no fue superado en ningún caso cuando se calculó eliminando cada vez uno de los ítems. La correlación entre las dos mitades del cuestionario fue de 0,88 y la fiabilidad según el estadístico de Guttman fue de 0,69. El rango de

Tabla 1. Características demográficas de la muestra y medidas de tendencia central obtenidas en los cuestionarios

\begin{tabular}{|c|c|c|c|}
\hline Variable & n (\%) & $\mathrm{M}(\mathrm{DT})$ & Mediana \\
\hline$\overline{\text { Edad }}$ & & $28,75(11,98)$ & \\
\hline De 19 a 24 años & $288(63,02)$ & & \\
\hline De 25 a 44 años & $82(17,94)$ & & \\
\hline De 45 a 60 años & $87(19,04)$ & & \\
\hline \multicolumn{4}{|l|}{ Sexo } \\
\hline Mujeres & $338(73,96)$ & & \\
\hline Hombres & $119(26,04)$ & & \\
\hline \multicolumn{4}{|l|}{ Profesión } \\
\hline Estudiantes Universidad & $291(63,67)$ & & \\
\hline Amas de casa & $31(6,78)$ & & \\
\hline Profesional o Técnico superior & $38(8,32)$ & & \\
\hline Profesional o Técnico medio & $33(7,22)$ & & \\
\hline Trabajador especializado & $64(14)$ & & \\
\hline \multicolumn{4}{|l|}{ Estado Civil } \\
\hline Soltero/a & $322(71,4)$ & & \\
\hline Casado/a & $118(25,8)$ & & \\
\hline Separado/a & $17(3,7)$ & & \\
\hline Amplificación Somatosensorial (SAS) & & $15,12(5,40)$ & 15 \\
\hline Conciencia Corporal Privada (CCP-BCQ) & & $7,19(2,91)$ & 7 \\
\hline Indice Witheley (IW- IBQ) & & $9,43(5,14)$ & 8 \\
\hline Escala de Preocupación por la Enfermedad & & $4,94(6,80)$ & 3 \\
\hline Hipocondría (Hs-MMPI) & & $8,77(5,05)$ & 8 \\
\hline STAI-Estado & & $18,70(10,09)$ & 18 \\
\hline STAI-Rasgo & & $20,67(9,66)$ & 20 \\
\hline Inventario Depresión Beck (BDI) & & $5,91(5,56)$ & 5 \\
\hline
\end{tabular}


correlaciones de cada ítem con la puntuación total en la escala osciló entre 0,38 y 0,65 . En cuanto al rango de las correlaciones entre las puntuaciones en cada uno de los ítems y la puntuación total corregida en la SAS, osciló entre 0,32 y 0,58 . En todos los casos, los niveles de significación estadística alcanzados fueron superiores a 0,001 .

En cuanto a la estructura interna de la SAS, la más adecuada e interpretable fue la de dos factores $(39,44 \%$ de varianza explicada total), cada uno de los cuales agrupó cinco variables y explicó un porcentaje de varianza similar $(19,80 \%$ y $19,64 \%$ ). En el primer factor se incluyeron los ítems $1,3,4,6$, y 9 , mientras que el segundo agrupó los cinco ítems restantes $(2,5,7,8$ y 10). En la Tabla 2 se presenta la estructura factorial obtenida. El contenido de los ítems del primer factor hace referencia, genéricamente, a una sensibilidad general hacia las señales interoceptivas del funcionamiento corporal, mientras que el segundo refiere más bien a una sensibilidad exagerada hacia estímulos molestos captados por los órganos sensoriales, ya se trate de estímulos provenientes del exterior (humo, ruidos, temperatura), o procedentes del propio cuerpo (dolor, contracciones de hambre).

Por lo que se refiere a las correlaciones
Spearman entre la puntuación total de la SAS y el resto de variables incluidas en el estudio, los resultados se ofrecen en la Tabla 3. Como puede observarse, la escala mantuvo correlaciones estadísticamente significativas con todas las variables. En el caso de las variables demográficas (edad estado civil, profesión y sexo), el coeficiente de correlación fue negativo, mientras que en las correlaciones con las variables medidas por cuestionarios, todos los coeficientes fueron de signo positivo. Con el fin de explorar más adecuadamente la naturaleza de estas relaciones, realizamos diversos análisis de varianza univariados (ANOVAs) o pruebas $t$ para muestras independientes, tomando siempre como variable dependiente la puntuación total en la SAS y como independiente/s las puntuaciones en cuestionarios $y / 0$ las variables sociodemográficas. En la Tabla 3 se ofrecen los valores obtenidos en cada caso, así como las puntuaciones medias detectadas en cada grupo de sujetos. En la primera columna de la tabla se presentan las puntuaciones medias en la SAS para cada uno de los grupos de sujetos en cada variable; la segunda columna presenta los resultados obtenidos tras la aplicación de pruebas $\mathrm{t}$ (dos grupos); la tercera columna recoge los resultados de los ANOVAs (más de dos grupos); la última

Tabla 2. Análisis factorial de la Escala de Amplificación Somatosensorial (SAS)

\begin{tabular}{rlrr}
\hline \multicolumn{1}{c}{ Item } & Factor 1 & Factor 2 \\
\hline 3. A menudo soy consciente de las diversas cosas que ocurren en mi cuerpo & $\mathbf{0 , 5 6}$ & $-0,18$ \\
4. Cuando me hago alguna magulladura, me la sigo notando durante mucho tiempo & $\mathbf{0 , 6 5}$ & $\mathbf{0 , 1 2}$ \\
1. El hecho de que alguien tosa, hace que yo tosa también & $\mathbf{0 , 7 0}$ & $-\mathbf{0 , 1 3}$ \\
6. Algunas veces puedo oír mi pulso o los latidos de mi corazón palpitando & & \\
& $\mathbf{0 , 5 5}$ & 0,28 \\
9. Realmente, hasta algo sin importancia, como una picadura de un insecto o & & \\
9incharme con una astilla, me molesta & $\mathbf{0 , 4 7}$ & $\mathbf{0 , 3 0}$ \\
2. No puedo soportar el tabaco, el humo o los factores contaminantes en el ambiente & $\mathbf{0 , 1 0}$ & $\mathbf{0 , 5 3}$ \\
5. Realmente los ruidos fuertes y repentinos me molestan & 0,23 & $\mathbf{0 , 5 3}$ \\
7. Odio tener demasiado calor o frío & 0,23 & $\mathbf{0 , 6 8}$ \\
8. Enseguida siento las contracciones de hambre en mi estómago & 0,28 & $\mathbf{0 , 5 4}$ \\
10. Tengo escasa tolerancia al dolor & 0,11 & $\mathbf{0 , 5 7}$ \\
& Varianza explicada & $19,80 \%$ & $19,64 \%$ \\
\hline
\end{tabular}


columna incluye los coeficientes de correlación (Spearman).

Como puede observarse, obtuvimos diferencias significativas en la puntuación total obtenida en la SAS según los diversos grupos establecidos. En el caso de los análisis de varianza y los subsiguientes post-hoc realizados, los resultados fueron los siguientes. Por lo que se refiere a la edad, las diferencias encontradas se debieron a que los sujetos más jóvenes alcanzaron puntuaciones más altas que los otros dos grupos, mientras que entre estos últimos no se detectaron diferencias. En cuanto a la profesión, los estudiantes universitarios y las amas de casa fueron quienes obtuvieron las pun- tuaciones más altas, diferenciándose en este sentido de los otros 3 grupos profesionales, que alcanzaron puntuaciones similares. En el caso del estado civil, las diferencias se obtuvieron entre los solteros y los otros dos grupos, siendo aquellos los que puntuaron más en la SAS.

En cuanto a las diferencias de género, las mujeres obtuvieron puntuaciones significativamente mayores que los hombres y cuando realizamos un ANOVA 2x3 con las variables Sexo y 3 Grupos de edad (19-24 años, 25-44 años, 45-60 años), detectamos efectos significativos tanto para la variable sexo como para la edad, además de para la interacción de ambas $(F=8,90 ; p<0,0001)$, lo que significa que

Tabla 3. Correlaciones entre la puntuación total de la SAS y el resto de variables socio-demográficas y de cuestionario, y diferencias en la SAS según grupos establecidos en tales variables

\begin{tabular}{|c|c|c|c|c|}
\hline Variable & $\begin{array}{l}\text { M (DT) } \\
\text { en SAS }\end{array}$ & $t$ & $\mathrm{~F}$ & $\begin{array}{l}r \text { con } \\
\text { SAS }\end{array}$ \\
\hline Edad & & & $11,08^{\star * \star}$ & $-0,25^{\star \star \star}$ \\
\hline De 19 a 24 años & $19,94(4,74)$ & & & \\
\hline De 25 a 44 años & $12,95(5,12)$ & & & \\
\hline De 45 a 60 años & $14,41(6.92)$ & & & \\
\hline Sexo & & $2,76^{\star \star \star}$ & & $-0,10^{\star}$ \\
\hline Mujeres & $15,53(5,15)$ & & & \\
\hline Hombres & $13,94(5,93)$ & & & \\
\hline Profesión & & & $8,02^{\star \star \star}$ & $-0,25^{\star \star *}$ \\
\hline Estudiantes Universidad & $16,06(4,71)$ & & & \\
\hline Amas de casa & $16,29(6,34)$ & & & \\
\hline Profesional o Técnico superior & $12,89(5,85)$ & & & \\
\hline Profesional o Técnico medio & $13(6,06)$ & & & \\
\hline Trabajador especializado & $12,92(6,23)$ & & & \\
\hline Estado Civil & & & $5,85^{\star \star}$ & $-0,19 \star \star \star$ \\
\hline Soltero/a & $15,68(4,91)$ & & & \\
\hline Casado/a & $13,77(6,57)$ & & & \\
\hline Separado/a & $13,72(3,28)$ & & & \\
\hline Conciencia Corporal Privada (CCP-BCQ) (a) & $18,15(4,68)$ vs. $12,59(4,60)$ & $12,73^{\star \star \star}$ & & $0,63^{\star \star \star}$ \\
\hline Indice Witheley (IW- IBQ) (a) & $17,03(5,12)$ vs. $13,87(5,25)$ & $6,33^{\star \star \star}$ & & $0,32^{\star \star \star}$ \\
\hline Escala de Preocupación por la Enfermedad (a) & $16,13(5,36)$ vs. $14,33(5,22)$ & $3,54^{\star \star \star}$ & & $0,21 * \star \star$ \\
\hline Hipocondría (Hs- MMPI) (a) & $16,71(5,09)$ vs. $13,36(5,20)$ & $6,92^{\star \star \star}$ & & $0,35^{\star \star \star}$ \\
\hline STAI-Estado (a) & $16,04(5,30)$ vs. $14,12(5,36)$ & $3,82^{\star \star \star}$ & & $0,20^{\star \star \star}$ \\
\hline STAI-Rasgo (a) & $16,24(5,27)$ vs. $13,81(5,26)$ & $4,92^{\star \star \star}$ & & 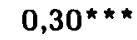 \\
\hline Inventario Depresión Beck (BDI) & $19,05(5,73)$ vs. $14,95(5,33)$ (b) & $3,18^{\star \star}$ & & $0,30^{\star \star \star}$ \\
\hline Grupo 1 (No depresión) & $14,44(5,19)$ & & & \\
\hline Grupo 2 (Depresión leve) & $17,36(5,45)$ & & & \\
\hline Grupo 3 (Depresión moderada) & $18,28(5,47)$ & & & \\
\hline Grupo 4 (Depresión grave) & $18,75(6,50)$ & & \multicolumn{2}{|c|}{$9,49^{\star \star \star}(\mathrm{c})$} \\
\hline
\end{tabular}

Nota: ${ }^{*} \mathrm{p}<0,05 ;{ }^{*} \mathrm{p}<0,001 ;{ }^{* \star} \mathrm{p}<0,0001$. (a) Para el establecimiento de los dos grupos el punto de corte fue la mediana del cuestionario; (b) El punto de corte para establecer dos grupos fue 18, el valor estimado como indicador de depresión clínica (más explicación en el texto); (c) El ANOVA se realizó sobre cuatro grupos (más explicación en el texto). 
las mujeres más jóvenes obtienen puntuaciones más elevadas que los hombres del mismo grupo etario.

Para analizar diferencias en la SAS según los cuestionarios, calculamos el valor de la mediana para cada uno (en la Tabla 1 se encuentran estos valores). Tomando los respectivos valores de la mediana como punto de corte establecimos dos grupos (puntuaciones elevadas versus bajas) en cada uno de los cuestionarios, excepción hecha del BDI que más adelante comentaremos, y calculamos entonces diversas pruebas $t$ sobre los valores alcanzados por cada grupo en la SAS. También en este caso obtuvimos diferencias significativas en la dirección esperada: todos los sujetos que obtuvieron puntuaciones altas en cada uno de los cuestionarios, diferían de los que las obtuvieron bajas en cuanto a su puntuación total en la escala de amplificación, ya que los primeros alcanzaron siempre valores más elevados en amplificación somatosensorial.

En el caso del Inventario de Beck para la Depresión, realizamos primero una prueba $t$, tomando el valor 18 como punto de corte, dado que se ha señalado que esta puntuación maximiza la exactitud diagnóstica del BDI (Chan, 1991; Rudd y Rajab, 1995), además de estar ampliamente consensuado para distinguir entre personas deprimidas y no deprimidas (Kendall, Hollon, Beck, Hammen e Ingram, 1987; Vázquez y Sanz, 1997). Según este criterio, nuestros resultados muestran que los deprimidos amplifican más que los no deprimidos sus sensaciones corporales. Realizamos un segundo análisis tomando en esta ocasión como criterio de agrupación los 4 puntos de corte establecidos por Beck y Steer (1993) para diferenciar entre ausencia de depresión o depresión mínima (0-9 puntos en BDI), depresión leve (10-16 puntos en BDI), depresión moderada (17-29 puntos en BDI) y depresión grave (30-63 puntos en BDI). También en este caso obtuvimos un valor de $\mathrm{F}$ significativo, que según los análisis post-hoc, se debe a que los tres grupos de depresión (leve, $\mathrm{n}=72$; moderada, $n=21$; y grave, $n=4$ ) alcanzaron una puntuación más alta en SAS que el grupo de sujetos no deprimidos $(n=360)$. En consecuencia, tanto si se toma como punto de corte en el BDI el primer valor (18), como si se establecen cuatro grupos según el criterio de Beck y Steer (1993), las personas que obtienen puntuaciones elevadas en este inventario difieren de las que puntúan por debajo del criterio en cuanto a su tendencia a amplificar sensaciones corporales inocuas.

Con el fin de averiguar si las variables estudiadas tenían capacidad predictiva sobre la puntuación en la SAS, se realizó un análisis de regresión múltiple (Pasos sucesivos), considerando la puntuación total en la escala SAS como variable dependiente y el resto de variables demográficas y de cuestionario como independientes. Solamente tres variables entraron en la ecuación de regresión. La primera de ellas fue la puntuación total en la escala CCP $\left(R^{2}=0,45\right.$; beta $=0,60$ ), la segunda fue la puntuación en ansiedad rasgo (STAI-R) $\left(R^{2}=\right.$ 0,03 ; beta $=0,15$ ), y la tercera fue la profesión $\left(R^{2}=0,01\right.$; beta $\left.=-0,12\right)$. En conjunto, las tres variables explicaban aproximadamente el $50 \%$ de la puntuación total en la SAS $\left(R^{2}=0,495\right)$.

Por último nos planteamos comprobar si existían diferencias entre los sujetos que obtenían puntuaciones altas, intermedias y bajas en la SAS con respecto a todas las variables consideradas. Para ello, establecimos tres grupos de personas, tomando como puntos de corte la media $\pm 1 \mathrm{DT}$ en la puntuación total de la SAS. Según este procedimiento, el $68 \%$ de los sujetos forman el grupo intermedio que agrupa a la mayoría de la población, mientras que el restante $26 \%$ se desglosa en dos grupos extremos: el de los que 
obtienen las puntuaciones más bajas (13\%) y el de los que obtienen las máximas puntuaciones (13\%). En el primer grupo se incluyeron los sujetos que obtuvieron una puntuación entre 1-9 $(n=63)$, en el intermedio los que puntuaron entre 10-20 $(n=330)$, y en el tercero los que alcanzaron puntuaciones entre 21-50 $(n=64)$ en la SAS. Sobre estos tres grupos realizamos ANOVAs para cada una de las variables de cuestionario y para la edad, con las correspondientes pruebas posthoc (Bonferroni) (Nota 1). Los resultados se resumen en la Tabla 4.
Como puede observarse los tres grupos de sujetos difirieron en cuanto a las puntuaciones obtenidas en los diversos cuestionarios (Conciencia Corporal Privada, Indice Witheley, Hipocondría (MMPI), Ansiedad Estado, Ansiedad Rasgo, y Depresión) y con respecto a su edad. En todos estos casos además, las diferencias fueron en la dirección que cabía esperar: cuanto más puntuaban en SAS (grupo 3) mayores puntuaciones alcanzaban también en los cuestionarios mencionados. Por lo que se refiere a la Escala de Preocupación por la Enfermedad también se

Tabla 4. ANOVAs para tres grupos de sujetos según su puntuación en la SAS

\begin{tabular}{|c|c|c|c|}
\hline Variable $^{\mathrm{B}}$ & $M(D T)$ & $\begin{array}{c}\mathrm{F}_{(2,456)} \\
\text { (post-hoc)* }\end{array}$ & $p<$ \\
\hline $\begin{array}{l}\text { Edad } \\
\text { Grupo } 1 \\
\text { Grupo } 2 \\
\text { Grupo } 3\end{array}$ & $\begin{array}{r}35,08(12,14) \\
27,82(11,58) \\
27,53(12,21) \\
\\
4,01(2,32) \\
7,19(2,42) \\
10,31(2,40)\end{array}$ & $\begin{array}{c}10,37 \\
1 \neq(2=3) \\
106,82\end{array}$ & 0,0001 \\
\hline $\begin{array}{l}\text { Indice Witheley (IW-IBQ) } \\
\text { Grupo } 1 \\
\text { Grupo } 2 \\
\text { Grupo } 3\end{array}$ & $\begin{array}{r}7,16(4,23) \\
9,28(4,82) \\
12,37(6,16)\end{array}$ & 17,88 & 0,001 \\
\hline $\begin{array}{l}\text { Escala de Preocupación por la Enfermedad } \\
\text { Grupo } 1 \\
\text { Grupo } 2 \\
\text { Grupo } 3\end{array}$ & $\begin{array}{l}3,18(4,23) \\
4,79(6,56) \\
7,40(9,18)\end{array}$ & $\begin{array}{c}6,31 \\
(1=2) \neq 3\end{array}$ & 0,002 \\
\hline $\begin{array}{l}\text { Hipocondría (Hs) MMPI } \\
\text { Grupo } 1 \\
\text { Grupo } 2 \\
\text { Grupo } 3\end{array}$ & $\begin{array}{r}5,83(4,31) \\
8,73(4,77) \\
11,75(5,46)\end{array}$ & $\begin{array}{c}23,70 \\
1 \neq 2 \neq 3\end{array}$ & 0,0001 \\
\hline $\begin{array}{l}\text { STAI-Estado } \\
\text { Grupo } 1 \\
\text { Grupo } 2 \\
\text { Grupo } 3\end{array}$ & $\begin{array}{l}15,30(10,11) \\
18,49(9,40) \\
23,25(11,92)\end{array}$ & $\begin{array}{c}10,48 \\
1 \neq 2 \neq 3\end{array}$ & 0,0001 \\
\hline $\begin{array}{l}\text { STAI-Rasgo } \\
\text { Grupo } 1 \\
\text { Grupo } 2 \\
\text { Grupo } 3\end{array}$ & $\begin{array}{l}15,01(9,07) \\
20,53(8,77) \\
26,96(11,04)\end{array}$ & $\begin{array}{c}26,95 \\
1 \neq 2 \neq 3\end{array}$ & 0,0001 \\
\hline $\begin{array}{l}\text { Inventario Depresión Beck } \\
\text { Grupo } 1 \\
\text { Grupo } 2 \\
\text { Grupo } 3\end{array}$ & $\begin{array}{l}2,93(3,30) \\
5,92(5,51) \\
8,79(6,08)\end{array}$ & $\begin{array}{c}18,82 \\
1 \neq 2 \neq 3\end{array}$ & 0,0001 \\
\hline
\end{tabular}

Nota: ${ }^{\circledR}$ Los grupos 1,2 y 3 se establecen sobre la base de la puntuación total en la SAS (véase el texto). Grupo 1= SAS 1-9; Grupo 2= SAS 10-20; Grupo 3= SAS 21-50. *Pruebas post-hoc. Los dígitos corresponden al número del grupo. $\neq$ diferentes entre sí; = iguales entre sí. 
mantuvo este patrón, pero en este caso los sujetos incluidos en los grupos 1 y 2 de la SAS no alcanzaron diferencias significativas en la mencionada EPE. Finalmente, los sujetos de los grupos 2 y 3 de la SAS no diferían en edad, pero en conjunto se diferenciaban del grupo 1 , cuya media de edad era mayor.

\section{DISCUSIÓN}

El propósito de este estudio ha sido poner a prueba la hipótesis de que la amplificación somato-sensorial constituye una fuente de diferencias individuales y por lo tanto, aunque puede constituir un elemento patogénico en la hipocondría, pueden detectarse también personas "amplificadoras» en la población general normal o sana. Esta hipótesis se puede derivar, en nuestra opinión, de los planteamientos de Barsky y su grupo en torno al concepto de amplificación. Para ello, analizamos en primer lugar las propiedades psicométricas de la Escala de Amplificación Somato-sensorial, un instrumento que según sus autores permite detectar la presencia del «estilo somático amplificador» (Barsky et al., 1990).

Los resultados obtenidos en el análisis de la consistencia interna de la escala fueron aceptables, por lo que puede decirse que la escala resulta ser una medida fiable de la tendencia a amplificar sensaciones corporales benignas. Según otros estudios, tanto en su versión inicial de 5 ítems (Barsky et al., 1988) como en la más reciente de 10 (Barsky et al., 1990), que es la que nosotras hemos utilizado, la escala ha mostrado una adecuada consistencia interna y fiabilidad test-retest. Así, por ejemplo, en el estudio de Barsky et al. (1990c) en el que utilizaron una muestra de pacientes obtenidos de una población médica general, la escala alcanzó una consistencia de 0,82 y una fiabilidad test-retest de 0,79 con un inter- valo temporal medio de 7 días. En otro estudio (Speckens, Spinhoven, Sloekers, Bolk y Hemert, 1995), si bien el valor de alfa no fue muy alto, especialmente para una muestra de pacientes de medicina general $(\alpha=0,61)$, la escala presentó una buena fiabilidad test-retest $(\alpha=0,87)$. En un estudio posterior de este mismo grupo (Speckens, Hemert, Spinhoven y Bolk, 1996), el valor de alfa en la población general $(n=185)$ fue de 0,71. Finalmente, en un estudio que realizamos con una muestra reducida de pacientes hipocondríacos y con trastorno de pánico (Martínez, Belloch y Botella, en prensa), el valor de $\alpha$ fue prácticamente similar al obtenido en este caso $(0,77$ vs. 0,79$)$. En definitiva, parece que nuestros resultados actuales se encuentran en la misma línea de los hallados por otros autores y utilizando diversos grupos de personas.

Sin embargo, por lo que se refiere a la estructura interna de la escala, nuestros resultados no son coincidentes con los publicados hasta la fecha. En nuestra muestra la amplificación se desglosa en dos factores, que difieren en cuanto al tipo de amplificación que recogen: en el primero de ellos se agrupan aspectos relacionados con lo que podría caracterizarse como sensibilidad genérica hacia los indicios interoceptivos, mientras que el segundo incluye sobre todo sensibilidad hacia estímulos exteriores que afectan el funcionamiento del cuerpo. Este resultado contrasta con el obtenido en el estudio antes mencionado de Speckens et al. (1996), en el que se encontró un único factor, y con el de Martínez et al. (en prensa) en el que se hallaron también dos factores pero con una composición ligeramente distinta a la encontrada en el presente trabajo. Estas diferencias son seguramente atribuibles a la procedencia y tamaño de la muestra (34 pacientes en el caso del trabajo de Martínez et al., frente a 457 personas sanas en nuestro caso), o bien a aspectos relacionados con 
procesos culturales, en el caso de los trabajos de Speckens y su grupo.

Por lo que se refiere a los resultados obtenidos tomando en consideración las variables socio-demográficas, constatamos que las mujeres amplifican más sus sensaciones corporales que los hombres, que los más jóvenes amplifican más que los mayores, y que lo mismo sucede con los estudiantes y amas de casa en comparación con el resto de grupos profesionales estudiados. Estos resultados son consistentes con los de otros trabajos por lo que se refiere al género. Por ejemplo en el estudio realizado también sobre población general de Speckens et al (1996), se constató que las mujeres obtenían puntuaciones más elevadas que los hombres en la medida de amplificación somatosensorial, aunque esta tendencia no se reprodujo en grupos de pacientes procedentes de un contexto médico. Sin embargo, en cuanto a la edad nuestros resultados discrepan de los obtenidos por Barsky, Frank, Cleary, Wyshak y Klerman (1991), ya que no encontraron diferencias en la medida de amplificación al comparar pacientes de medicina general de distintas edades.

En cuanto a la validez concurrente de la SAS, nuestros resultados fueron satisfactorios. La escala presentó correlaciones significativas con cuatro instrumentos que valoran diversos aspectos relacionados con hipocondría: la Escala de Conciencia Corporal Privada, la Escala de Hipocondría del MMPI, el Índice Witheley y la Escala de Preocupación por la Enfermedad. Además, los sujetos que obtuvieron las puntuaciones más elevadas en estos indicadores de hipocondría, diferían de los que puntuaron bajo en cuanto a su amplificación: los primeros amplifican más sus sensaciones corporales inocuas que los segundos. Finalmente, los análisis de regresión mostraron que la puntuación en Conciencia Corporal Privada es, con mucho, el mejor pre- dictor de la amplificación, ya que por sí sola explicó el $45 \%$ de esta última. La conclusión que, desde nuestro punto de vista, se puede extraer de estos resultados es que la amplificación se relaciona de forma importante con indicadores de hipocondría. Ello avala la utilidad de la escala y, por lo tanto, del constructo teórico en el que se sustenta, la amplificación somático-sensorial, como un indicador fiable de actitudes hipocondríacas en la población general.

Por otro lado, la SAS presentó relaciones importantes con ansiedad rasgo y ansiedad estado (evaluadas con el STAI), y con depresión (valorada con el BDI). Estos resultados se asemejan parcialmente a los obtenidos en estudios previos. En el ya mencionado de Barsky et al. (1988), la amplificación se asoció significativamente a la sintomatología depresiva, la ansiedad y la presencia de síntomas somáticos en una muestra de pacientes con trastornos respiratorios. Por lo que respecta a la asociación entre amplificación y ansiedad puede tomarse como un elemento a favor de quienes opinan que es necesario reconceptuar la hipocondría como un trastorno de ansiedad (por ejemplo, Salkovskis y Clark, 1993). Sin embargo, si nos atenemos al planteamiento que el propio Barsky realiza de la amplificación (Barsky, 1992), ésta no se plantea como en elemento asociado exclusivamente a la hipocondría, sino que debe conceptuarse como un síntoma inespecífico de otros trastornos mentales que cursan con sintomatología física, como es el caso de la ansiedad y la depresión. La diferencia, según este autor, radicaría en el role patogénico que la amplificación juega en la hipocondría, frente a otro mucho más inespecífico en trastornos como los mencionados. De hecho, la SAS también correlacionó significativamente con depresión y el tamaño del coeficiente obtenido fue igual que para la ansiedad rasgo $(0,30)$ y superior que el obtenido ara ansiedad estado $(0,20)$. 
Por lo que se refiere a los resultados obtenidos a partir de los puntos de corte que establecimos para la SAS, hay que resaltar, en primer lugar, que las puntuaciones en la SAS mostraron una distribución perfectamente ajustada a la normalidad estadística.

En segundo término, parece interesante señalar aquí que, en un estudio previo realizado con pacientes diagnosticados de hipocondría (Martínez et al., en prensa), la puntuación media alcanzada en la SAS fue de $28,52 \pm 7,88$, con un rango que osciló entre 16 y 43 . Solamente dos pacientes obtuvieron una puntuación total en la escala inferior a 21 , lo que los hubiera situado en el grupo intermedio del presente estudio. El resto obtuvo puntuaciones superiores a 21 , que ha sido nuestro punto de corte para establecer el grupo 3, es decir, el de los amplificadores. Si consideramos estos datos en términos de percentiles (véase la Nota 1), todos los pacientes se hubieran situado por encima del percentil 80. A la luz de todos estos datos, y aun siendo conscientes de que la muestra del estudio al que nos estamos refiriendo era pequeña (17 pacientes), pensamos que podemos afirmar con cierta seguridad que puntuaciones totales en la SAS superiores a 21 indican una elevada tendencia, cercana a lo psicopatológico, a amplificar las sensaciones corporales. Lógicamente, sería necesario realizar estudios específicos para dilucidar si, además, esta tendencia puede conceptuarse como un marcador de vulnerabilidad a la hipocondría.

Nuestro grupo de "amplificadores», es decir, los que puntuaron por encima de 21 en amplificación (o los que se situaron en el percentil 90) difirieron ampliamente de los no amplificadores (los otros dos grupos) en todas las variables incluidas en este estudio, excepción hecha de la edad, ya que los amplificadores máximos (grupo 3) no difieren en este parámetro de la mayoría de la población (grupo 2, que agrupa al $68 \%$ de la muestra) . Este dato es explicable teniendo en consideración la distribución de edad de nuestra muestra: la mayoría eran jóvenes, por lo que no es de extrañar que fueran ellos, frente a los más mayores, quienes obtuvieran las puntuaciones más elevadas. En nuestra opinión, ello matiza, al menos en parte, nuestros resultados a este respecto. Es posible que, puesto que la gran mayoría de nuestra muestra sea joven, la población de más edad se halle poco representada en nuestro estudio.

Por otra parte, es interesante constatar que, ateniéndonos a los puntos de corte ampliamente aceptados para realizar una aproximación a la sintomatología depresiva sobre la base del Inventario de Beck, nuestro grupo de amplificadores no presentaba sintomatología depresiva o la presentaba en grado mínimo. Tampoco alcanzaron los valores medios estimados en ansiedad para la población diagnosticada con trastornos de ansiedad, según la adaptación española del STAI. Si se consideran estos resultados conjuntamente con los que ya hemos comentado para el caso del BDI y el STAI, en donde constatamos que las personas que obtienen las mayores puntuaciones en estos instrumentos, son las que a su vez más puntúan en amplificación, podemos aventurar la hipótesis de que los individuos ansiosos y deprimidos amplifican más sus sensaciones corporales que los no ansiosos y no deprimidos, pero sin embargo no se produce el patrón inverso: los que más amplifican, no están necesariamente deprimidos ni presentan trastornos de ansiedad. Esto significaría, en nuestra opinión, que la amplificación es una variable diferente de la ansiedad y la depresión. $\mathrm{O}$, si se prefiere, que la presencia de amplificación no es sinónimo de (o no indica necesariamente la presencia de) éstas dos últimas. Sin embargo, es muy probable que niveles altos de ansiedad y presencia de sintomatología 
depresiva se asocien con una mayor tendencia a amplificar síntomas corporales inocuos. Todo esto apoyaría la ya comentada opinión de Barsky acerca de la amplificación como una variable presente en muchos trastornos, pero no asimilable a éstos.

En conclusión, la SAS es una escala fiable y válida para evaluar el constructo teórico en el que se sustenta, la amplificación somato-sensorial. Además, se halla presente en la población general, y adquiere la forma de una dimensión continua a lo largo de la cual existen amplias diferencias individuales en edad, depresión, ansiedad, y diversos indicadores aceptados de hipocondría. Por último, pensamos que puede resultar de utilidad para valorar la predisposición a adoptar actitudes hipocondríacas, lo que sin duda requerirá el diseño de investigaciones específicamente dirigidas a examinar este importante aspecto.

\section{REFERENCIAS BIBLIOGRÁFICAS}

Ballester, R., y Botella, C. (1993). Perfil de Conducta de Enfermedad en pacientes con crisis de angustia. Análisis y Modificación de Conducta, 19, 233-265.

Barsky, A.J. (1979). Patients who amplify bodily sensations. Annals of International Medicine, 91, 63-70.

Barsky, A.J. (1992). Amplification, somatization, and the somatoform disorders. Psychosomatics, 33, 28-34.

Barsky, A.J., Goodson, J.D., Lane, R.S., y Cleary, P.D. (1988). The amplification of somatic symptoms. Psychosomatic Medicine, 50, 510-519.

Barsky, A.J., y Klerman, G.L. (1983). Overview: hypochondriasis, bodily complaints, and somatic styles. American Journal of Psychiatry, 140, 273-283.

Barsky, A. J., y Wyshak, G. (1990). Hypochondriasis and somatosensory amplification. British Journal of Psychiatry, 157, 404-409.
Barsky, A.J., Wyshak, G., y Klerman, G.L. (1990). The somatosensory amplification scale and its relationship to hypochondriasis. Journal of Psychiatric Research, 24, 323-334.

Beck, A.T., Rush, A.J., Shaw, B.F., y Emery, G. (1979). Cognitive Therapy of Depression. New York: Guilford Press (Traducción castellana en Bilbao, Desclée de Brower, 1983).

Chan, D.W. (1991). The Beck Depression Inventory: What difference does the Chinese version make? Psychological Assessment, 3, 616-622.

Hathaway, S.R., y McKinley, J.C. (1967). Minnesota Multiphasic Personality Inventory. New York: The Psychological Corporation (Adaptación Española: Madrid: TEA, 1988). Jiménez, M.A., Castañeiras, C., Martínez, P., Ayllón, E. y Belloch, A. (1997). Validación de la Escala de Preocupaciones por la Enfermedad. I Congreso de la Asociación Española de Psicología Clínica y Psicopatología. Madrid, Abril 1997.

Kendall, P.C., Hollon, S.D., Beck, A.T., Hammen, C.L. e Ingram, R.E. (1987). Issues and recommendations regarding the use of the Beck Depression Inventory. Cognitive Therapy and Research, 11, 289-299.

Martínez, M.P., Belloch, A., y Botella, C. (en prensa). Somatosensory amplification in hypochondriasis and panic disorder. Clinical Psychology and Psychotherapy.

Miller, L.C., Murphy, R., y Buss, A.H. (1981). Consciousness of body: private and public. Journal of Personality and Social Psychology, 4, 397-406.

Pilowski, I., y Spence, N.D. (1983). Manual for the Illness Behavior Questionnaire. Australia: Department of Psychiatry, Adelaide University.

Rudd, M.D., y Rajab, M.H. (1995). Specificity of the Beck Depression Inventory and the confounding role of comorbid disorders in a clinical sample. Cognitive Therapy and Research, 19, 51-68.

Salkovskis, P., y Clark, D.M. (1993). Panic disorder and hypochondriasis. Advances in Behaviour Research and Therapy, 15, 23-48.

Seisdedos, N. (1988). Cuestionario de Ansiedad Estado-Rasgo. Adaptación Española. Madrid: TEA. 
Speckens, A.E., Spinhoven, P., Sloekers, P.P., Bolk, J.H., y Hemert, A.M. (1995). A Validation study of the Whiteley Index, the Illness Attitude Scales, and the Somatosensory Amplication Scale in general medical and general practice patients. Journal of Psychosomatic Research, 40, 95-104.

Speckens, A.E., Hemert, A.M., Spinhoven, P., y Bolk, J.H. (1996). The diagnostic and prognostic significance of the Whiteley Index, the Illness Attitude Scales and the Somatosensory Amplification Scale. Psychological Medicine, 26, 1085-1090.

Spielberger, C.D., Gorsuch, R.L., y Lushene, R.E. (1970). STAI, Manual for the StateTrait Anxiety Inventory (Self Evaluation Questionnaire). Palo Alto, California: Consulting Psychologists Press.

Vázquez, C., y Sanz, J. (1997). Fiabilidad y valores normativos de la versión española del Inventario para la Depresión de Beck de 1978. Clínica y Salud, 8, 403-422.

Wesner, R.B., y Noyes, R. (1991). Imipramine an effective treatment for illness phobia. Journal of Affective Disorders, 22, 43-48.

\section{NOTAS}

(1) Además de este procedimiento, calculamos también la distribución de la muestra utilizando percentiles. Los resultados de formación de grupos fueron prácticamente los mismos, ya que por debajo del percentil 10 se situaron los sujetos con puntuaciones entre 18, y por encima del percentil 90 los que obtuvieron puntuaciones superiores a 21 (amplitud intercuartil = 6; IC $95 \%=14,62-15,61$ ). Dada la equivalencia de resultados, optamos por el primer procedimiento para los subsiguientes análisís de comparación entre grupos. 